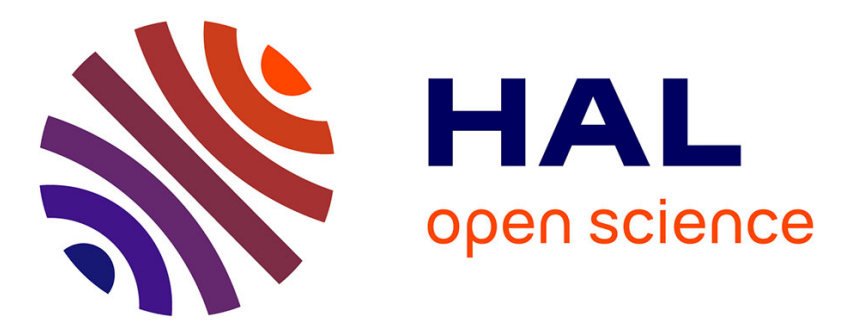

\title{
Vision-based assistance for wheelchair navigation along corridors
}

\author{
François Pasteau, Alexandre Krupa, Marie Babel
}

\section{To cite this version:}

François Pasteau, Alexandre Krupa, Marie Babel. Vision-based assistance for wheelchair navigation along corridors. IEEE Int. Conf. on Robotics and Automation, ICRA'14, Jun 2014, Hong-Kong, Hong Kong SAR China. hal-00949188

\section{HAL Id: hal-00949188 \\ https://hal.inria.fr/hal-00949188}

Submitted on 19 Feb 2014

HAL is a multi-disciplinary open access archive for the deposit and dissemination of scientific research documents, whether they are published or not. The documents may come from teaching and research institutions in France or abroad, or from public or private research centers.
L'archive ouverte pluridisciplinaire HAL, est destinée au dépôt et à la diffusion de documents scientifiques de niveau recherche, publiés ou non, émanant des établissements d'enseignement et de recherche français ou étrangers, des laboratoires publics ou privés. 


\title{
Vision-based assistance for wheelchair navigation along corridors
}

\author{
François Pasteau ${ }^{1}$, Alexandre Krupa ${ }^{2}$ and Marie Babel ${ }^{1}$
}

\begin{abstract}
In case of motor impairments, steering a wheelchair can become a hazardous task. Typically, along corridors, joystick jerks induced by uncontrolled motions are source of wall collisions. This paper describes a vision based assistance solution for safe indoor semi-autonomous navigation purposes. To this aim, the control process is based on a visual servoing process designed for wall avoidance purposes. As the patient manually drives the wheelchair, a virtual guide is defined to progressively activate an automatic trajectory correction. The proposed solution does not require any knowledge of the environment. Experiments have been conducted over corridors that present different configurations and illumination conditions. Results demonstrate the ability of the system to smoothly and adaptively assist people during their motions.
\end{abstract}

\section{INTRODUCTION}

The sudden loss of motor and/or cognitive capabilities as well as chronic or degenerative impairment can induce permanent handicaps. Disabling conditions have thus to be carefully considered to preserve or provide people independence and autonomy. In particular, in [1], it has been shown that autonomy and dignity are strongly linked. The ability to freely move remains then a fundamental need for well-being and living well at home. Moreover, maintaining this capacity prevents from affecting the mental condition.

In this context, assistive robotics for mobility purposes are closely in relation with personal living assistance major issues. Autonomous or semi-autonomous wheelchair navigation has been widely studied in the literature [2]. A semi-autonomous navigation system refers to a cooperation between the user and the robotic system. The idea is then to provide an aid for navigation that improves the ease to steer the wheelchair and thus leads to reduce fatigue sensation. To maximize the acceptability of the assistance solution, this assistance has to be adaptively activated only when necessary and to be deactivated as soon as the user wants to act by himself [3]. As a consequence, the control process has to be designed as a man-in-the-loop scheme where the user remains the leader of the navigation process. Different solutions have been already proposed in this context. They typically rely on the partial or global knowledge of the environment [4]. We here aim at designing a control system that performs in an unknown environment.

In addition, difficulties appear during long-term driving. Depending on the handicap, steering a wheelchair along a corridor can become a difficult task especially when corridors remain narrow enough to induce an uncomfortable sensation

\footnotetext{
${ }^{1}$ François Pasteau and Marie Babel are with INSA Rennes, IRISA and Inria Rennes, France, marie.babel at irisa.fr

${ }^{2}$ Alexandre Krupa is with Inria Rennes and IRISA, France, alexandre.krupa at inria.fr
}

in navigation [2]. In [5], authors present a semi-autonomous navigation process that relies on an innovative interface based on a Muscle-Computer Interface (MCI). The control process simply reduces the velocity of the wheelchair in case of rotation. In our case, we aim at designing a wall avoidance task that uses visual feedback. To insure a widespread use of such a system, we target low-cost architecture, including a single monocular camera. A dedicated visual servoing framework is then designed, that fuses both the manual control and a vision based wall avoidance task. Our previous work [6] has demonstrated the ability of a visual servoing framework to provide full-autonomous navigation solution throughout a corridor. In contrast, the present paper presents an innovative semi-autonomous navigation framework that combines visual servoing and manual control. The main idea is to correct the trajectory during the manual control by gradually activating a visual servoing task when the wheelchair gets too close to the corridor walls. The proposed approach allows the user to still keep manual control on the degrees of freedom (DOF) that does not compromise a safe navigation.

This paper is organized as follows. The section II details the modelling of the robotized wheelchair. In section III, the required visual features are defined. Control task by visual servoing for wall avoidance purposes is then described in section IV. The semi-autonomous navigation system results then of the fusion of the manual control and the visual servoing (section V). Visual servoing experiments are shown in section VI and results are presented and discussed.

\section{MODELING}

We consider the wheelchair as a six-wheel robot that moves on a horizontal plane. Two differentially actuated wheels are located at the middle of the robot body. Additional two passive caster front wheels and two passive caster rear wheels are also required. A wheelchair can be modelled as a unicycle robot, thus matching nonholonomous constraints. The two control variables related to the wheelchair are then the translation velocity $u$ along its forward/backward direction and the angular (steering) velocity $\omega$. Figure 1 depicts the different cartesian frames considered in this modelling. $\mathcal{F}_{g}\left(O, x_{g}, y_{g}, z_{g}\right)$ represents the world frame and $\mathcal{F}_{r}\left(P_{O}, x_{r}, y_{r}, z_{r}\right)$ is a frame of the wheelchair attached on the middle of the segment formed by the centers of the two differentially actuated wheels. We define $\mathcal{F}_{c}\left(C, x_{c}, y_{c}, z_{c}\right)$ as the camera frame that is rigidly fixed to the wheelchair, where $C$ represents the optical center. The image frame is defined by $\mathcal{F}_{I}\left(I_{0}, X, Y\right)$ where $I_{0}$ is the center point of the image. Relatively to $\mathcal{F}_{g}$, the robot state coordinates 


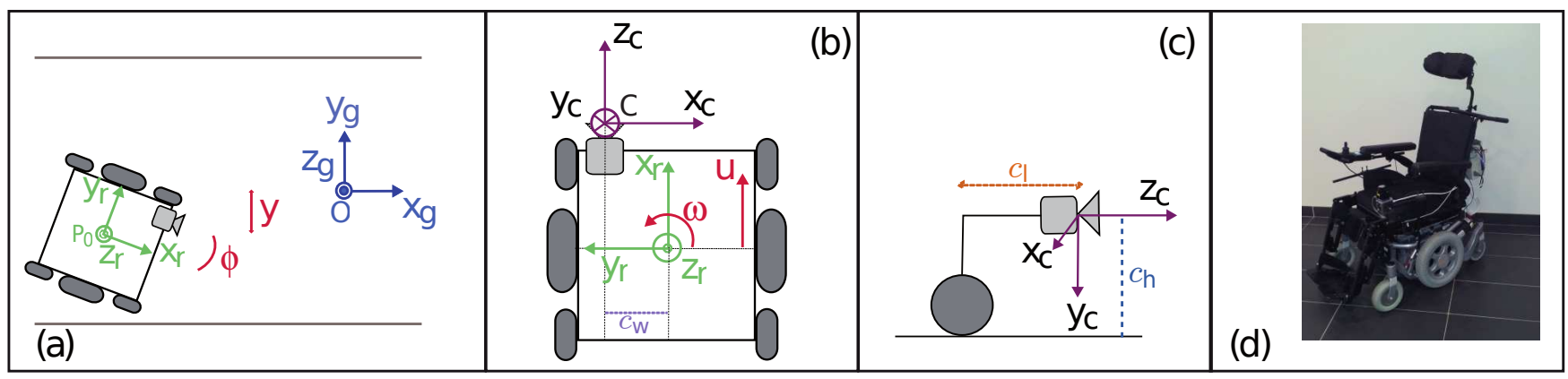

Fig. 1. World, robot and camera frames. (a) Top view of the wheelchair with related global and robot frames. (b) Top view with robot and camera frame relative positions. (c) Simplified side view. (d) Robotic platform.

are given by $\mathbf{q}=(x, y, \phi)$ where $x$ and $y$ denote the Cartesian position of the robot, and $\phi \in[-\pi, \pi]$ corresponds to the angle between the driving direction of the wheelchair and the $\mathrm{x}$-axis of the world coordinate frame. In short, $\phi$ corresponds to the heading angle defined as the positive counterclockwise orientation of the wheelchair with respect to $x_{g}$ axis. The optical center of the camera is positioned on the wheelchair in such a way that we observe a translation vector ${ }^{c} \mathbf{t}_{r}=\left(c_{w}, 0,-c_{l}\right)$. The distance between the floor and the camera optical center is equal to $c_{h}$.

The rotation matrix ${ }^{c} \mathbf{R}_{r}$ that models the fixed orientation of the camera frame relatively to the robot frame is given by

$$
{ }^{c} \mathbf{R}_{r}=\left[\begin{array}{ccc}
0 & -1 & 0 \\
0 & 0 & -1 \\
1 & 0 & 0
\end{array}\right] .
$$

The robot jacobian ${ }^{r} \mathbf{J}_{r}$ expressed in the robot frame is equal to

$$
{ }^{r} \mathbf{J}_{r}=\left[\begin{array}{cccccc}
1 & 0 & 0 & 0 & 0 & 0 \\
0 & 0 & 0 & 0 & 0 & 1
\end{array}\right]^{T}
$$

Let the control inputs be $\mathbf{u}=(u, \omega)$. The kinematic model of the camera expressed in the world frame is given by

$$
\left\{\begin{array}{l}
\dot{x}=u \cos \phi-c_{l} \dot{\phi}, \\
\dot{y}=u \sin \phi-c_{w} \dot{\phi}, \\
\dot{\phi}=\omega .
\end{array}\right.
$$

\section{VISUAL FEATURES}

In this paper we propose a semi-autonomous navigation mode that will assist disabled people driving a wheelchair in a corridor. The objective is to provide at the control level a virtual guide like a crash barrier that will help the user to follow a corridor without hitting the lateral walls. The assistance mode we here propose consists of sharing the 2 control inputs $\mathbf{u}=(u, \omega)$ of the wheelchair between a visual servoing task for wall avoidance and the manual control of handicapped people.

In [6] a full-autonomous corridor following task was performed thanks to an image-based visual servoing that regulates a set of 2 visual features $\mathbf{s}=\left(x_{f}, \theta_{m}\right)$ to desired values. These visual features are respectively the $\mathrm{x}$-axis coordinate $x_{f}$ of the vanishing point $f$ detected in the image and the angle $\theta_{m}$ of the 2D projection of the median line of

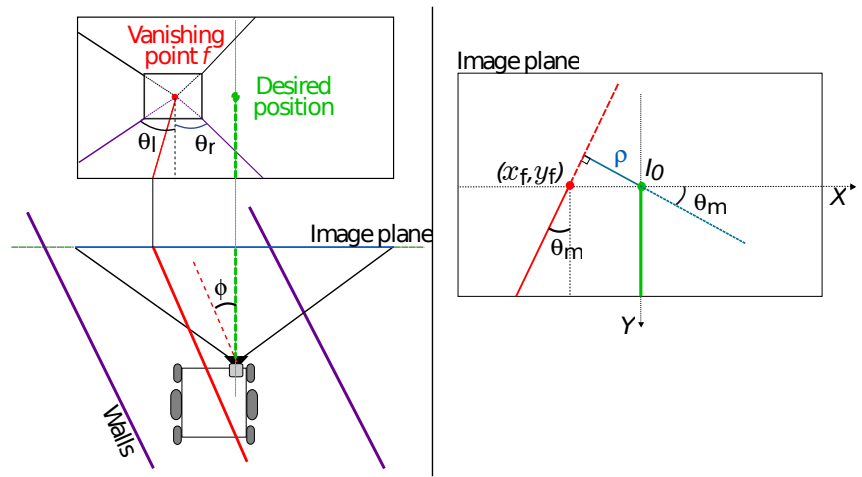

Fig. 2. $\quad \theta$ and $x_{f}$ visual features

the corridor into the image as illustrated in Figure 2. This 2D projection corresponds to the straight line parametrized by $\left(\rho_{m}, \theta_{m}\right)$ where

$$
\rho_{m}=x_{f} \cos \theta_{m}+y_{f} \sin \theta_{m}
$$

and

$$
\theta_{m}=\arctan \left(\frac{1}{2}\left(\tan \left(\theta_{l}\right)+\tan \left(\theta_{r}\right)\right)\right),
$$

with $\theta_{r}$ and $\theta_{l}$ being respectively the orientations of the left and right straight lines related to the bottom of the walls (see Figure 2). As demonstrated in [6], if a constant velocity forward motion $u^{*}$ is applied to the wheelchair, its orientation is ensured to be parallel to the corridor walls if $x_{f}$ has converged to zero. Moreover the lateral position of the wheelchair reaches the middle of the corridor width when $\theta_{m}$ has converged also to zero. A visual control scheme was therefore presented in [6] to servo the steering angle $\omega$ in such a way to minimize these two visual features to zero when a constant forward velocity is applied to the wheelchair.

In this work, we consider the same visual features for the wall avoidance task since they provide an efficient description of the orientation of the robot and its lateral position with respect to the corridor walls. In our previous work [6], only $\omega$ was controlled by visual servoing with a constant velocity $u$ and no interaction of the user was taken in account in the wheelchair steering. In contrast, we propose in the next section a visual control scheme sharing all the 
degrees of freedom between the user and a wall avoidance task. We invite the reader to refer to [6] for the image processing framework required to extract the visual features in real-time.

\section{WALL AVOIDANCE TASK BY VISUAL SERVOING}

To insure that the wheelchair does not hit the corridor walls, we propose to gradually activate the regulation of the visual features $\mathbf{s}=\left(x_{f}, \theta_{m}\right)$ to the desired values $\mathbf{s}^{*}=(0,0)$ when they leave safe intervals that we fixed, namely $x_{f} \in$ $\left[x_{f}^{s-}, x_{f}^{s+}\right]$ and $\theta_{m} \in\left[\theta_{m}^{s-}, \theta_{m}^{s+}\right]$, in order they go back inside these safe intervals. This concept of an interval that triggers the visual servoing was introduced in [7] and used in [8] to insure the visibility of an organ section during remote ultrasound tele-echography. By adapting this framework to our wall avoidance task, we propose the following visual control law aiming at keeping the visual features inside their interval:

$$
\mathbf{u}=-\lambda\left(\mathbf{H J}_{s}\right)^{+} \mathbf{H e}
$$

where $\lambda>0$ is the control gain, $\mathbf{e}=\mathbf{s}-\mathbf{s}^{*}$ is the visual error and $\mathbf{J}_{s}$ is the image Jacobian that links the variation of the visual features to the robot control input such that $\dot{\mathbf{s}}=\mathbf{J}_{s} \mathbf{u}$. This image Jacobian was determined in [6] and was formulated as follows:

$$
\mathbf{J}_{s}=\mathbf{L}_{s}{ }^{c} \mathbf{W}_{r}{ }^{r} \mathbf{J}_{r}
$$

with $\mathbf{L}_{s}$ being the interaction matrix that relates the variation of the visual features to the camera velocity screw. Let $\mathrm{c}=$ $\cos \left(\theta_{m}\right)$ and $\mathrm{s}=\sin \left(\theta_{m}\right) . \mathbf{L}_{s}$ is defined by

$\mathbf{L}_{s}=\left[\begin{array}{cccccc}0 & 0 & 0 & x_{f} y_{f} & -1-x_{f}^{2} & y_{f} \\ \lambda_{\theta_{m}} \mathrm{c} & \lambda_{\theta_{m}} \mathrm{~s} & -\lambda_{\theta_{m}} \rho_{m} & -\rho_{m} \mathrm{c} & -\rho_{m} \mathrm{~s} & -1\end{array}\right]$

with $\lambda_{\theta_{m}}=\cos \left(\theta_{m}\right) / c_{h}$. The matrix ${ }^{c} \mathbf{W}_{r}$ is the velocity screw transformation that links the camera velocity (expressed in $\mathcal{F}_{c}$ ) to the robot velocity screw (expressed in $\left.\mathcal{F}_{r}\right)$ :

$$
{ }^{c} \mathbf{W}_{r}=\left[\begin{array}{cc}
{ }^{c} \mathbf{R}_{r} & {\left[{ }^{c} \mathbf{t}_{r}\right]_{\times}{ }^{c} \mathbf{R}_{r}} \\
\mathbf{0}_{3 \times 3} & { }^{c} \mathbf{R}_{r}
\end{array}\right]
$$

with []$_{x}$ the skew matrix expression. By combining (2) and (7) in (6), we immediately obtain:

$\mathbf{J}_{s}=\left[\begin{array}{cc}0 & 1+x_{f}^{2} \\ -\lambda_{\theta_{m}} c_{l} \mathbf{c}+\lambda_{\theta_{m}} w \rho_{m}+\rho_{m} \mathbf{s} & -\lambda_{\theta_{m}} \rho_{m}\end{array}\right]=\left[\begin{array}{c}\mathbf{J}_{x_{f}} \\ \mathbf{J}_{\theta_{m}}\end{array}\right]$

In the control law (5), $\mathbf{H}=\operatorname{Diag}\left(h_{f_{x}}, h_{\theta_{m}}\right)$ is a diagonal matrix that weights the visual error where $h_{f_{x}} \in[0 ; 1]$ and $h_{\theta_{m}} \in[0 ; 1]$ are varying weights respectively associated to the visual features $f_{x}$ and $\theta_{m}$. A null weight means that the related visual feature is not regulated by the visual servoing. The matrix $\mathbf{H}$ allows then to add or remove any visual feature in the control law when desired and can totally deactivate the visual servoing when $\mathbf{H}$ is null. In order to gradually activate the wall avoidance task when a visual feature leaves its safe interval, we propose to define the weights by the following smooth function:

$h_{x_{f}}\left(x_{f}\right)=\left\{\begin{array}{cl}\left(1-\cos \left(\pi \frac{x_{f}-x_{f}^{s-}}{x_{f}^{-}-x_{f}^{s-}}\right)\right) / 2 & \text { if } x_{f}^{-} \leq x_{f} \leq x_{f}^{s-} \\ 0 & \text { if } x_{f}^{s-}<s_{f}<x_{f}^{s+} \\ \left(1-\cos \left(\pi \frac{x_{f}-x_{f}^{s+}}{x_{f}^{+}-x_{f}^{s+}}\right)\right) / 2 & \text { if } x_{f}^{s+} \leq x_{f} \leq x_{f}^{+} \\ 1 & \text { otherwise }\end{array}\right.$

where $\left[x_{f}^{-}, x_{f}^{+}\right]$is a tolerated interval including the safe one whose fixed limits should never be overcame thanks to visual servoing. A similar expression for $h_{\theta_{m}}$ can also be proposed: corresponding function evolution is shown in Figure 3. We

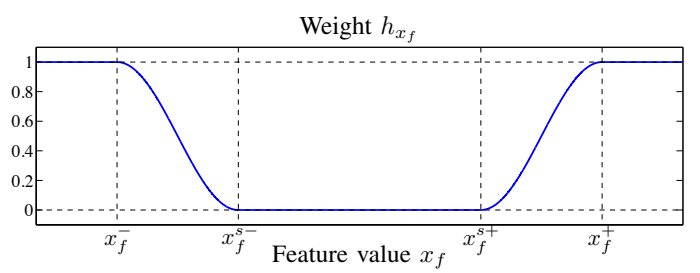

Fig. 3. Weighting function $h_{x_{f}}$ defined for feature $x_{f}$. The weight is null in the safe interval and increases smoothly up to 1 at the borders of the tolerated interval $\left[x_{f}^{-}, x_{f}^{+}\right]$(a similar function is used to define the weight $h_{\theta_{m}}$ with intervals $\left[\theta_{m}^{-}, \theta_{m}^{+}\right]$and $\left.\left[\theta_{m}^{s-}, \theta_{m}^{s+}\right]\right)$.

can note that each weight is null in the safe interval and increases up to 1 if the related feature comes close to the tolerated interval limits. In this way, the wall avoidance task is gradually activated when the weight related to the visual feature is increasing.

The three conditions to obtain a continuous behaviour of the control law (5) are that $\mathbf{J}_{s}, \mathbf{H}$ and the pseudo-invese of $\mathbf{H J}_{s}$ remain continuous. The two first conditions are valid according to the form of (8) and the weight definition (9). However, the pseudo-inverse is not continuous since the rank of $\mathbf{H J}_{s}$ could switch from null if all features are in their safe intervals to 1 when only one feature leaves its interval or 2 (full rank) when the two features are outside their safe intervals. To avoid discontinuities in the control we propose therefore to replace like in [8] the Moore-Penrose pseudoinverse operator ${ }^{+}$by the continuous pseudo-inverse operator $\bigoplus \mathbf{H}$ introduced in the framework of varying-feature-set [9]. This operator allows the inversion of a matrix $\mathbf{J}$ weighted by a diagonal matrix $\mathbf{H}$ by applying the following definition:

$$
\mathbf{J}^{\oplus \mathbf{H}}=\sum_{\mathcal{P} \in \mathfrak{B}(k)}\left(\prod_{i \in \mathcal{P}} h_{i}\right)\left(\prod_{i \notin \mathcal{P}}\left(1-h_{i}\right)\right) \mathbf{J}_{\mathcal{P}}^{+}
$$

where $\mathbf{J}$ is a matrix of size $(k \times n), \mathbf{H}$ is a diagonal activation matrix of size $(k \times k)$ whose components $\left(h_{i}\right)_{i \in[1 \ldots k]}$ are included in the interval $[0,1] \cdot \mathfrak{B}(k)$ is the set of all the combinations formed by the integers belonging between 1 and $k$ (for example $\mathfrak{B}(2)=\{\emptyset,\{1\},\{2\},\{1,2\}\}$ ). $\mathcal{P}$ is any element of this set and $\mathbf{J}_{\mathcal{P}}=\mathbf{H}_{0} \mathbf{J}$ with $\mathbf{H}_{0}$ being a $(k \times k)$ diagonal matrix whose component $(i, i)$ is equal to 1 if $i \in \mathcal{P}$ and to 0 otherwise. For more details, all the theoretical bases 
including the proof of continuity of this inversion operator are presented in [9]. By applying this operator for our wall avoidance task (with $k=2$ ), the continuous inversion of the image jacobian $\mathbf{J}_{s}$ activated by the weight matrix $\mathbf{H}$ is given by:

$$
\begin{aligned}
\mathbf{J}_{s}^{\oplus \mathbf{H}}= & +h_{x_{f}}\left(1-h_{\theta_{m}}\right)\left[\begin{array}{c}
\mathbf{J}_{x_{f}} \\
\mathbf{0}_{1 \times 2}
\end{array}\right]^{+} \\
& +\left(1-h_{x_{f}}\right) h_{\theta_{m}}\left[\begin{array}{c}
\mathbf{0}_{1 \times 2} \\
\mathbf{J}_{\theta_{m}}
\end{array}\right]^{+} \\
& +h_{x_{f}} h_{\theta_{m}} \mathbf{J}_{s}^{+}
\end{aligned}
$$

We can note that if all the weights of $\mathbf{H}$ are equal to 1 then the matrix $\mathbf{J}_{s}^{\oplus \mathbf{H}}$ is exactly equal to $\left(\mathbf{H} \mathbf{J}_{s}\right)^{+} \mathbf{H}$ and we have the same equality if all the weights are null. The control law (5) can therefore be replaced by the following law insuring the wall avoidance task with a continuous behaviour:

$$
\mathbf{u}=-\lambda \mathbf{J}_{s}^{\oplus} \mathbf{H} \mathbf{e}
$$

\section{FUSION OF AVOIDANCE TASK AND MANUAL CONTROL}

In practice, the avoidance task will gradually be activated when at least one feature leaves its safe interval. That means that only the degree(s) of freedom (DOF) required to regulate the activated feature/(s) is/(are) constrained by the visual task. It is therefore possible to manually control the remaining DOF thanks to the well-known redundancy formalism [10]. Moreover, when all the features have gone back in their safe intervals, all the DOF are available for the manual control since the visual servoing is automatically deactivated if $\mathbf{H}=\mathbf{0}$. This also means that the desired features $\mathbf{s}^{*}$ (that we arbitrary fixed in the center of the intervals) will never be reached: this is not a problem since the objective of the visual servoing is only to bring them back in their safe intervals. The wall avoidance task can therefore be fused with a secondary task by adding in (12) a second term as follows:

$$
\mathbf{u}=-\lambda \mathbf{J}_{s}^{\oplus} \mathbf{H} \mathbf{e}+\mathbf{P}_{\oplus} \mathbf{u}_{o p}
$$

where $\mathbf{u}_{o p}$ is an arbitrary vector used for performing a secondary task and $\mathbf{P}_{\oplus}=\mathbb{I}_{2}-\mathbf{J}_{s}^{\oplus} \mathbf{H} \mathbf{J}_{s}$ is the projection operator presented in [11]. This operator projects the component of $\mathbf{u}_{o p}$ onto the null space of the main task in order that the secondary one does not disturb the main one that has higher priority. To fuse the wall avoidance task with the wheelchair manual control, we apply the vector $\mathbf{u}_{o p}=$ $\left(u_{o p}, \omega_{o p}\right)$, where $u_{o p}$ and $\omega_{o p}$ are respectively the references of the forward/backward operated translation velocity and operated angular (steering) velocity transmitted by the user thanks to the wheelchair 2-axes joystick.

\section{EXPERIMENTAL RESULTS}

\section{A. Experimental setup}

In these experiments, the mobile robot is based on an off-the-shelf Penny and Giles wheelchair adapted to robotic use using RoS middleware [12]. The wheelchair is equipped with one forward looking Raspberry PI camera module with $f o v=85^{\circ}$ field of view [13]. The characteristics of the video stream from the camera correspond to a frame width of 808 pixels and a frame height of 480 pixels and a framerate of 15 frames per second. The camera and the mechanical system were coarsely calibrated with $c_{h}=0.64 \mathrm{~m}, c_{l}=0.38 \mathrm{~m}$, $c_{w}=0.32 \mathrm{~m}$. Prior to the feature extraction, images are rectified against the distortions due to the camera lens.

Visual feature extraction as well as control law computation use the ViSP software [14]. Computation is performed on a Core i7 laptop connected to the wheelchair using Ethernet network. With such a hardware configuration, a 30 ms latency is to be expected between the user command $u_{o p}$, $\omega_{o p}$ received from the joystick and the computed command $u, \omega$ received by the wheelchair.

For ground truth estimation and visualisation purposes, the wheelchair has been equipped with a laser range finder. This laser range finder is used neither in the visual feature extraction process nor in the control law. It then only acts as a validation tool.

To be able to perform experiments using the control law proposed in this paper, some parameters needs to be determined (i.e. $x_{f}^{+}, x_{f}^{s+}, x_{f}^{-}, x_{f}^{s-}, \theta_{m}^{+}, \theta_{m}^{s+}, \theta_{m}^{-}$and $\theta_{m}^{s-}$ ).

$x_{f}^{+}, x_{f}^{s+}, x_{f}^{-}, x_{f}^{s-}$ are directly dependent to the field of view of the camera. In our setup, the maximum and minimun possible values of $x_{f}$ are respectively equal to $\tan ($ fov $/ 2) \approx 0.916$ and $-\tan ($ fov $/ 2) \approx-0.916$. Under these constraints, we chose $x_{f}^{+}=0.9$ and $x_{f}^{-}=-0.9$ to ensure the visibility of the vanishing point. As the visual feature extraction algorithm needs to detect both floor/wall boundary lines, we chose $x_{f}^{s+}=0.3$ and $x_{f}^{s-}=-0.3$, to ensure the visibility of these features.

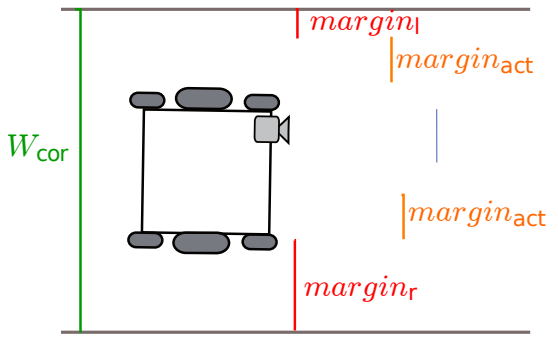

Fig. 4. Margin definition

$\theta_{m}^{+}, \theta_{m}^{s+}, \theta_{m}^{-}$and $\theta_{m}^{s-}$ are directly dependent to the width of the corridor used during the experimentation. The width of the corridor $W_{c o r}$ can be estimated during the feature extraction process using $W_{c o r} \approx c_{h}\left(\tan \left(\theta_{l}\right)+\tan \left(\theta_{r}\right)\right)$. Then by defining margin $_{l}$ and margin $_{r}$ being the wanted margin between the walls and the camera and margin $_{a c t}$ as depicted in figure 4 , we can determine :

$$
\begin{aligned}
\theta_{m}^{+} & =\arctan \left(\frac{W_{\text {cor }}-2 \operatorname{margin}_{r}}{2 c_{h}}\right) \\
\theta_{m}^{s+} & =\arctan \left(\frac{W_{\text {cor }}-2 m \operatorname{margin}_{r}-2 \text { margin }_{\text {act }}}{2 c_{h}}\right)
\end{aligned}
$$




$$
\begin{aligned}
\theta_{m}^{-} & =\arctan \left(\frac{2 \operatorname{margin}_{l}-W_{c o r}}{2 c_{h}}\right) \\
\theta_{m}^{s-} & =\arctan \left(\frac{2 \operatorname{margin}_{l}+2 \text { margin }_{a c t}-W_{c o r}}{2 c_{h}}\right)
\end{aligned}
$$

In our experiments, we choose margin $_{l}=0.15 \mathrm{~m}$, margin $_{r}=0.60 \mathrm{~m}$ and margin $_{\text {act }}=0.30 \mathrm{~m}$ and $W_{\text {cor }}$ is estimated using the camera at initialisation and is kept constant for the rest of the experiments.

The experimentations took place in three different corridors.

\section{B. Results of semi-autonomous navigation}

Figure 5 and 6 respectively show the variation of the feature weights $h_{x_{f}}$ and $h_{\theta_{m}}$ during the experimentation. The relationship of $h_{x_{f}}$ as function of $x_{f}$ and $h_{\theta_{m}}$ as function of $\theta_{m}$ have been defined by (9).

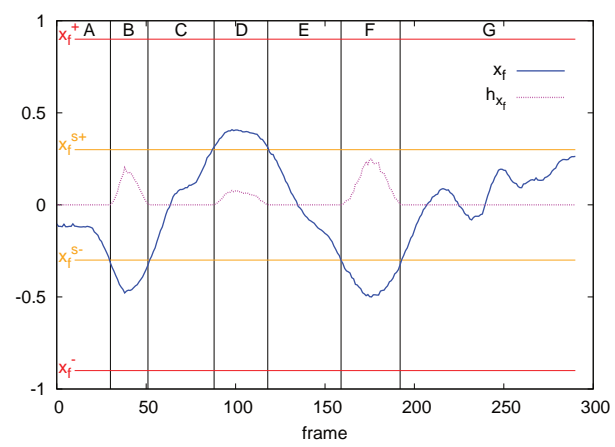

Fig. 5. Evolution of $h_{x_{f}}$

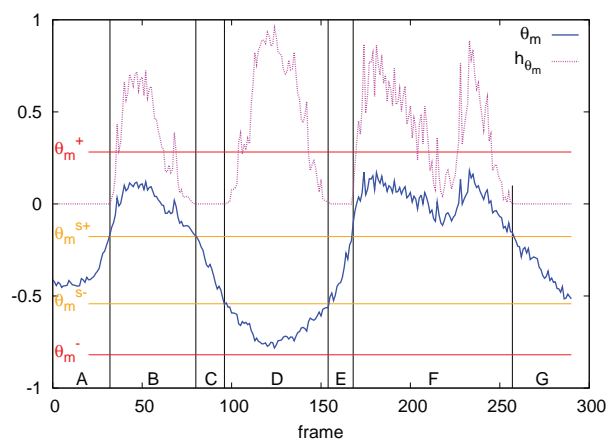

Fig. 6. Evolution of $\theta_{m}$

In parts $\mathrm{A}, \mathrm{C}, \mathrm{E}$ and $\mathrm{G}$ of figure $5, x_{f}$ remains in the $\left[x_{f}^{s-}, x_{f}^{s+}\right]$ interval and therefore $h_{x_{f}}$ remains equal to 0 . In the other parts of the figure, $x_{f}$ crosses either the $x_{f}^{s+}$ or $x_{f}^{s-}$ boundaries and $h_{x_{f}}$ becomes positive. On figure 6 , the same behavior can be observed with respectively $\theta_{m}, \theta_{m}^{s+}$ and $\theta_{m}^{s-}$. We can observe that during the experimentation, $x_{f}$ and $\theta_{m}$ were respectively forced by the visual servoing to remain in the interval $\left[x_{f}^{s-}, x_{f}^{s+}\right]$ and $\left[\theta_{m}^{s-}, \theta_{m}^{s+}\right]$ as expected.

Figure 7 presents the evolution of the different parameters of the control law during the experimentation. First, the top figure shows the evolution of $h_{x_{f}}$ and $h_{\theta_{m}}$, where the middle one concerns $u$ and $u_{o p}$ and finally the bottom one concerns $\omega$ and $\omega_{o p}$.
Figure 8 presents outputs of the wheelchair camera together with visualization of the position of the wheelchair in the corridor from the laser range finder at different key points during the experimentation. On each camera output, the blue/green line represents the estimated middle line of the corridor and the cross the estimated position of the vanishing point. The color of the line (cross) refers to the value of $h_{\theta_{m}}\left(h_{x_{f}}\right)$, green when $h_{\theta_{m}}=0\left(h_{x_{f}}=0\right)$ and blue when $h_{\theta_{m}}>0\left(h_{x_{f}}>0\right)$. The red and orange lines correspond to the boundary of $\theta_{m}$ according to (9). On each output of the laser range finder, the position of the wheelchair has been depicted in red with an arrow as forward direction.
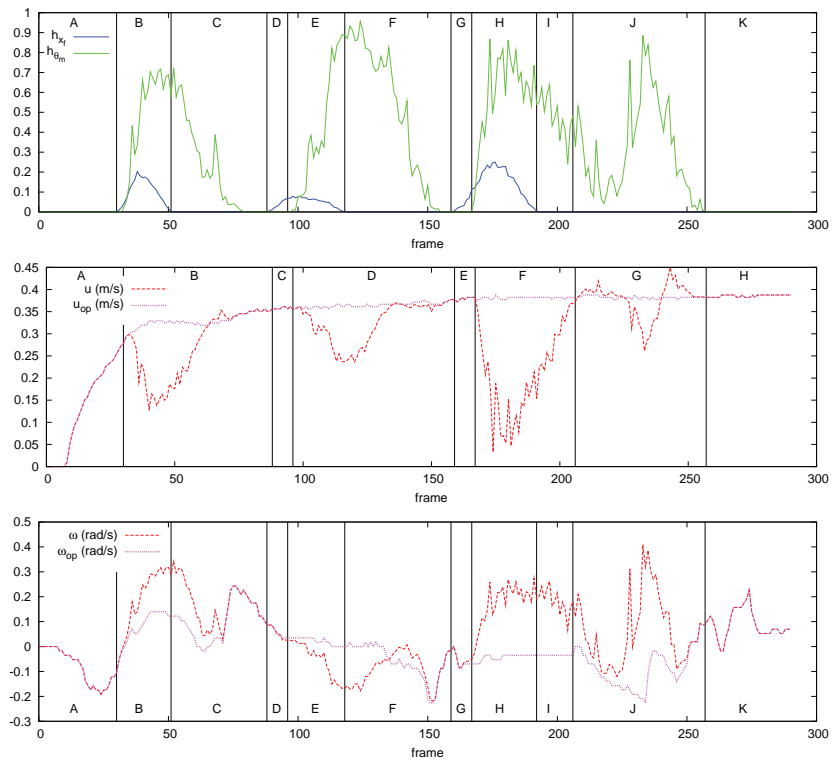

Fig. 7. Evolution of $h_{x_{f}}, \theta_{m}, u$ and $\omega$

In figure 7 , in parts $\mathrm{A}$ and $\mathrm{K}, h_{x_{f}}=0$ and $h_{\theta_{m}}=0$, we can then observe that $u=u_{o p}$ and $\omega=\omega_{o p}$. The user has then full control of the wheelchair in both translation and rotation. In figure 8 , frame 15 corresponds to part $\mathrm{A}$ situation. We can observe that the vanishing point is nearly in the middle of the image and that the middle line of the corridor is located between the orange line. On the laser range finder, we can observe that the wheelchair is in the middle of the corridor with a low orientation angle. In such a case, there is no risk of collision with the wall, therefore the user has full control on the wheelchair.

In figure 7, in parts $\mathrm{C}, \mathrm{F}, \mathrm{I}$ and $\mathrm{J}, h_{x_{f}}=0$ and $h_{\theta_{m}}>0$, leading then to $u \neq u_{o p}$ and $\omega \neq \omega_{o p}$. As shown in figure 8 , in part $C$ of frame 69 , the wheelchair is close to the right wall with a low orientation angle. There is then a risk of collision if the user tries to turn right. Therefore, the control law is activated and filters $u_{o p}$ and $\omega_{o p}$ to avoid wall collision.

In figure 7, in parts $\mathrm{B}, \mathrm{E}$ and $\mathrm{H}, h_{x_{f}}>0$ and $h_{\theta_{m}}>0$. We can then observe that $u \leq u_{o p}$ and $\omega \neq \omega_{o p}$. As seen in figure 8 , in part $\mathrm{E}$ frame 118, the wheelchair is close to the left wall and oriented toward the wall. There is an imminent risk of collision. The translation velocity $u$ is reduced to 


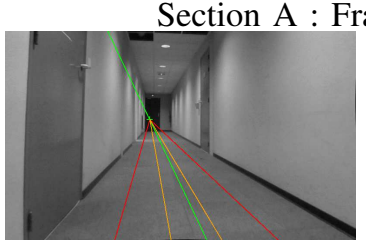

a) Wheelchair camera Section $\mathrm{E} \cdot \mathrm{F}$

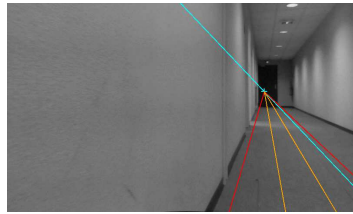

e) Wheelchair camera

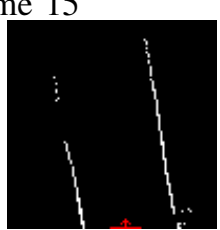

b) Ground truth

118

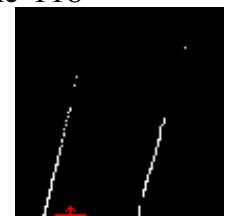

f) Ground truth

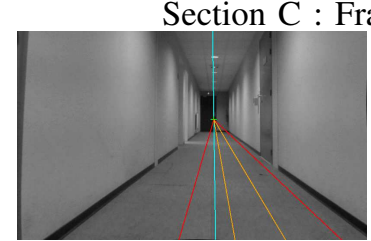

c) Wheelchair camera Section $\mathrm{G} \cdot$ Frame

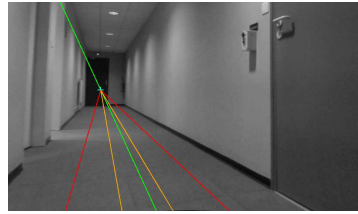

g) Wheelchair camera

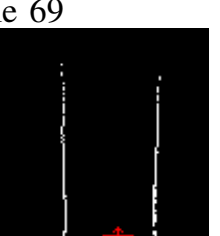

d) Ground truth 160

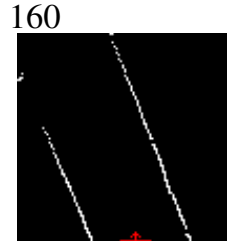

h) Ground truth

Fig. 8. Wheelchair camera frames and ground truth during the experimentation

avoid collision and the rotation velocity $\omega$ is forced to a negative value to get further from the wall.

In figure 7, in sections D and $\mathrm{G}, h_{x_{f}}>0$ and $h_{\theta_{m}}=0$, we observe that $u=u_{o p}$ and $\omega \neq \omega_{o p}$. From (8), $u=u_{o p}$ is to be expected as $u$ does not impact the vanishing point position $x_{f}$. As seen in figure 8 , in part $G$ frame 160 , the vanishing point coordinate $x_{f}$ is on the right side of the camera output. If the vanishing point gets too close to the border of the image, the visual feature extraction process may not have the required information. Therefore $\omega$ is servoed to avoid such a problem.

Additional results can be found on http://www.irisa.fr/lagadic/team/Marie.Babel-eng.html.

\section{CONCLUSION}

Preserving the autonomy and the mobility is essential for disabled people well-being. In this paper, we proposed a semi-autonomous system designed for safe wheelchair navigation along corridors. This control system relies on the combination of two tasks: first the manual steering and second wall avoidance task obtained by a dedicated visual based servoing approach. The idea is then to correct the trajectory indicated by the patient by servoing only the necessary degrees of freedom.

This visual servoing process is based on both the vanishing point and wall plane detection. A smooth transition from manual driving to assisted navigation is obtained thanks to an adapted weighting function, thus avoiding discontinuities that can lead to unpleasant experience. Results clearly show the ability of the approach to provide an efficient solution for wall avoiding purposes. Additional experiment sessions are planned with volunteers in order to evaluate the quality of experience.

We are currently working on a second complementary task, based on the same principle, which would include doorway passing.

\section{ACKNOWLEDGMENT}

This work is supported by APASH Oseo/Région Bretagne project and Inria Large-scale initiative action Personally
Assisted Living. The authors would like to thank Daniel Guillard and Luc Le Pape from Ergovie and Eric Bazin from INSA.

\section{REFERENCES}

[1] L. Nordenfelt, Dignity in Care for Older People. Wiley-Blackwell, 2009.

[2] A. Kokosy, T. Floquet, G. Howells, H. Hu, M. Pepper, and C. Donz, "SYSIASS An Intelligent Powered Wheelchair," in International Conference on Systems and Computer Science (ICSCS2012), 2012.

[3] B. Krieg-Brückner, D. Crombie, B. Gersdorf, A. Jüptner, M. Lawo, C. Mandel, A. B. Martínez, T. Röfer, and C. Stahl, "Challenges for indoor and outdoor mobility assistance," Technik für ein selbstbestimmtes Leben, 2012.

[4] P. Viswanathan, J. Little, A. K. Mackworth, and A. Mihailidis, "Adaptive navigation assistance for visually-impaired wheelchair users," in Proceedings of the IROS 2011 Workshop on New and Emerging Technologies in Assistive Robotics, 2011.

[5] F. Auat Cheein, N. Lopez, C. Soria, F. di Sciascio, F. Lobo Pereira, and R. Carelli, "Slam algorithm applied to robotics assistance for navigation in unknown environments," Journal of NeuroEngineering and Rehabilitation, vol. 7, no. 1, p. 10, 2010.

[6] F. Pasteau, M. Babel, and R. Sekkal, "Corridor following wheelchair by visual servoing," in IEEE/RSJ Int. Conf. on Intelligent Robots and Systems, IROS'2013, Tokyo, Japan, November 2013.

[7] O. Kermorgant and F. Chaumette, "Combining ibvs and pbvs to ensure the visibility constraint," in IEEE/RSJ Int. Conf. on Intelligent Robots and Systems, IROS'11, San Francisco, USA, September 2011, pp. 2849-2854.

[8] T. Li, O. Kermorgant, and A. Krupa, "Maintaining visibility constraints during tele-echography with ultrasound visual servoing," in IEEE Int. Conf. on Robotics and Automation, ICRA'12, Saint Paul, USA, May 2012, pp. 4856-4861.

[9] N. Mansard, A. Remazeilles, and F. Chaumette, "Continuity of varying-feature-set control laws," IEEE Trans. Autom. Control, vol. 54, no. 11, pp. 2493-2505, December 2009.

[10] C. Samson, B. Espiau, and M. Le Borgne, Robot control: The task function approach. Oxford University Press, 1991.

[11] N. Mansard, O. Khatib, and A. Kheddar, "A unified approach to integrate unilateral constraints in the stack of tasks," vol. 25, no. 3, pp. 670-685, 2009.

[12] M. Quigley, K. Conley, B. P. Gerkey, J. Faust, T. Foote, J. Leibs, R. Wheeler, and A. Y. Ng, "Ros: an open-source robot operating system," in ICRA Workshop on Open Source Software, 2009.

[13] R. pi fundation, "Raspberry Pi An ARM GNU/Linux box for $\$ 25$. Take a byte!" http://www.raspberrypi.org/, 2012, [Online; accessed 1September-2013].

[14] E. Marchand, F. Spindler, and F. Chaumette, "ViSP for visual servoing: a generic software platform with a wide class of robot control skills," IEEE Robotics and Automation Magazine, vol. 12, no. 4, pp. 40-52, 2005. 